\title{
A Mathematical Model for the Eradication of Anopheles Mosquito and Elimination of Malaria
}

\author{
Atanyi Yusuf Emmanuel (Corresponding Author) \\ Department of Mathematics, Federal University of Lafia, Nasarawa State, Nigeria \\ Email: abamayeni@gmail.com
}

\author{
Abam Ayeni Omini \\ Department of Mathematics, Federal University of Lafia, Nasarawa State, Nigeria
}

\author{
Article History \\ Received: December 8, 2019 \\ Revised: January 3, 2020 \\ Accepted: January 10, 2020 \\ Published: January 12, 2020 \\ Copyright (C) 2020 ARPG \& \\ Author \\ This work is licensed under \\ the Creative Commons \\ Attribution International \\ (c) (6) \\ Commons Creativ \\ Commons At \\ License 4.0
}

\begin{abstract}
A mathematical model to eliminate malaria by breaking the life cycle of anopheles mosquito using copepods at larva stage and tadpoles at pupa stage was derived aimed at eradicating anopheles pupa mosquito by introduction of natural enemies "copepods and tadpoles" (an organism that eats up mosquito at larva and pupa stage respectively). The model equations were derived using the model parameters and variables. The stability analysis of the free equilibrium states was analyzed using equilibrium points of Beltrami and Diekmann's conditions for stability analysis of steady state. We observed that the model free equilibrium state is stable which implies that the equilibrium point or steady state is stable and the stability of the model means, there will not be anopheles adult mosquito in our society for malaria transmission. The ideas of Beltrami's and Diekmann conditions revealed that the determinant and trace of the Jacobian matrix were greater than zero and less than zero respectively implying that the model disease free equilibrium state is stable. Hence, the number of larva that transforms to pupa is almost zero while the pupa that develop to adult is zero meaning the lifecycle is broken at the larva and pupa stages with the introduction of natural enemy. Maple was used for the symbolic and numerical solutions.
\end{abstract}

Keywords: Anopheles; Copepods; Malaria; Mosquito; Tadpoles.

\section{Introduction}

Malaria is one of the high priority tropical diseases in the sub-Saharan Africa in general and Nigeria in particular. Although there has been many efforts to eradicate malaria from the world over a long period of time, yet it remains a complex disease. It affects human health and continues to be one of the most serious vector borne diseases worldwide. Malaria, an endemic disease is transmitted through three possible media namely, malaria parasite, human host and anopheles mosquito. The female Anopheles mosquito feeds on human blood. This has resulted to the death of over two million people yearly in the Sub Saharan Africa, with one third of the victims as children particularly in Nigeria.

Mosquito is a common flying insect found around the world with approximately 3,500 species. For the purpose of this work, the research will be narrowed down to the female genius of anopheles mosquito of the approximately 430 species. The Anopheles go through four stages in their life cycle. The egg, larva, pupa and adult. The first three stages are aquatic lasting up to 5-14 days depending on the species and the ambient temperature. The adult stage which is the final stage is when the female anopheles mosquito acts as malaria vector. The adult female can live up to a month but most probably do not live more than 1-2 weeks in nature. This work focuses on a mathematical model to eliminate malaria by breaking the life cycle of Anopheles mosquito using copepods at larva stage and tadpoles at pupa stage. This study focused on anopheles mosquito a part of the malaria transmission cycle. The way to solve the malaria problem is to intervene the medium. This work is going to introduce the use of natural enemy as a predator which depends largely on the number of host or prey attacked or consumed to break the life cycle of the anopheles mosquito. The suitable environmental conditions for various behaviors of anopheles mosquito such as resting, swarming, oviposition, biting and feeding are examined [1].

Mathematical models provide a sound understanding of risk and planning for control in heterogeneous environments, especially when the models are based on the ecology of the vector population and a sound understanding of the entomological parameters relevant for transmission [2]. The researches conducted by Killeen, et al. [2] and Macdonald [3] states that Mathematical models also played important roles in understanding the epidemiology of malaria and other infectious disease; that Mathematical Models also provided concise quantitative description of complicated nonlinear processes and a method for relating the process of infection in individual to the incidence of disease or infection in a population overtime which important insight have come by the introduction of natural enemies to break the life cycle of anopheles mosquito at larva stage and pupa stage thus, reducing or 
eradicating mosquito [4]. This introduction of natural enemies reduces malaria by vector that bites. They work by reducing the intensity of malaria transmission or eradicating malaria. The classification of a natural enemy as a predator or parasite depends largely on the number of prey or host attacked or consumed the reproduction strategy and other details of the system in which there are many similarities in the characteristics of the natural enemy and in the properties of the model to study them. Some predators, such as copepods and predatory mosquitoes, have been suggested for most mosquito control.

The World Health Organization [5] realized that the global eradication of malaria was impossible for a variety of reasons and the control focus shifted to the control of deadly diseases [5]. Since the idea of eradicating mosquitoes was not realistic, the efforts were directed towards the reduction and management of their population below the threshold that would cause diseases [6]. More also, for the first time in Africa an entomological study went beyond the Conventional practice of determining parity and survival rates of field collected adult Anopheles mosquitoes but, also related these variables to duration of Plasmodium sporogeny and estimated the expectation of infective life. Hence blood seeking female mosquitoes was collected in Ilorin, Nigeria, from January, 2005 to December, 2006 [7]. Coetzee, et al. [1] studied thattThe Anopheles gambiae population in Ilorin is dominated by older mosquitoes with high survival rate thereby, suggesting a high vector potential for the species in the area [8]. This information on the survival ratio of Anopheles gambiae in relation to malaria transmission would enhance the development of a more focused and informed vector control interventions [1,9].

\subsection{Statement of the Problem}

Malaria has been noted by WHO's "Roll Back Malaria" as the major cause of death in our society. To curb this, this research seeks to derive and implement a mathematical model that will break the life cycle of Anopheles mosquito by introducing Natural Enemies. The equilibrium state of the model would be obtained, the stability criteria will be discussed using equilibrium point, the idea of Beltrami and Diekmann with some threshold result.

\subsection{Aim and Objectives of the Study}

The research work is aimed at presenting a mathematical model to eliminate malaria by breaking the life-cycle of anopheles mosquito using copepods at the larva stage and tadpoles at pupa stage. In order to achieve this, the following objectives will be considered.

i. To introduce natural enemies, copepods and tadpoles to the life-cycle of anopheles Mosquitoes at larva and pupa stage.

ii. To derive the model equations to break the life-cycle of anopheles mosquito at larva and pupa stage.

iii. To determine the stability analysis of the disease free equilibrium state of the model using equilibrium point, Beltrami's and Diekmann's conditions.

iv. To obtain graphical presentation of the result using maple software on the effect of introducing two natural enemies (copepods and tadpoles) on both larva and pupa stage.

\section{Review of Related Literature}

Research conducted by Jepson, et al. [10] measured the length of each of the three stages (egg, larva and pupa) of mosquitoes in different breeding habitat using the equation

$$
M=1 /(L e+L i+L p)
$$

where; $M$ is the maturation rate of mosquitoes. $L_{e}$ is the period of egg stage.

$\mathrm{L}_{\mathrm{i}}$ is the period of larva $\left(\mathrm{L}_{1}-\mathrm{L}_{4}\right)$ stages. $\mathrm{Lp}$ is the period of pupa stage.

Diekmann, et al. [11] determined the basic reproduction number $\left(\mathrm{R}_{0}\right)$ using the equation

$$
F=\frac{\partial F_{1}\left(X_{0}\right)}{\partial x j}, V=\frac{\partial V_{i}\left(X_{0}\right)}{\partial x j} \quad \ldots \quad \ldots . \quad \ldots
$$

Where $\mathrm{i}, \mathrm{j}=1 \ldots \ldots \ldots \mathrm{m}$ and where $\mathrm{X}_{0}$ is the disease free equilibrium. $\mathrm{FV}^{-1}$ gives the rate at which infected individuals in $\mathrm{xj}$ produces new infection in $\mathrm{X}_{\mathrm{i}}$.

The works of Smith and Mckenzie [9] on a journal article titled: the Statics and Dynamics of Malaria infection in Anopheles mosquito reveals the following;

i. The survivorship and life span of anopheles mosquito is the proportion of a cohort mosquito that survive at age $\mathrm{A}$, given by $\lambda A=e^{-g a}$, where $e^{-g a}$ is the probability that an individual mosquito survives one day.

The average life span of mosquitoes according to Smith and Mckenzie [9] is given by

$$
\int_{0}^{\infty} \operatorname{gA} \lambda(A) \partial A=\frac{1}{g}, \quad \ldots \quad \ldots \quad \ldots
$$

where $\mathrm{g} \lambda(\mathrm{A})$ is the proportion of mosquito that dies at age $\mathrm{A}, \mathrm{g}=$ force of mortality, $\mathrm{A}=$ age, $\frac{1}{\mathrm{~g}}=$ the average mosquito life span.

ii. The Human Feeding Stability Index and Human Blood Index (HBI) since a mosquito lives, $\frac{1}{\mathrm{~g}}$ days and bites human once everyday. Where $\mathrm{S}=$ The stability index which is the number of bites given by a mosquito after it has become infectious, $\mathrm{a}=$ expected number of bites and $\mathrm{a}=\mathrm{Qf}$ where $\mathrm{Q}=$ proportion of bites taken on human and $\mathrm{f}=$ mosquito feeding rate. Human Blood Index according to Smith and Mckenzie [9] is given by

$$
\int_{0}^{\infty} \eta(A) d A / \int_{0}^{\infty} \lambda(A) d A=\frac{a}{a+g}
$$


Where $\eta(A) \lambda=$ the proportion of mosquito in a population that has survived to age $\mathrm{A}$ and bitten a human being. Note that the proportion of feed mosquitoes $\frac{f}{f+g}$ can be derived in a similar way, assuming a $=\mathrm{f}$ thus the Human Blood Index (HBI) is a simple function of the mosquito life span and human feeding rate. It can be understood as a ratio of two waiting times; the waiting time to either the first human bite or death $\frac{1}{a+g}$ and the waiting time to the first human bite among surviving mosquitoes $\frac{1}{a}$.

iii. Proportion of infected mosquitoes according to Smith and Mckenzie [9] is given by

$$
\int_{0}^{\infty} v(A) \lambda(A) \frac{d A}{\lambda(A)}=\frac{a c X}{g+a c X} \quad \ldots \quad \ldots .
$$

Thus the proportion of infected mosquitoes is a ratio of two waiting times: the waiting time to either death or infection, $\frac{1}{g}+a c X$ and the waiting time to infection among surviving mosquitoes $\frac{1}{a c X}, V(A)=1-e^{-a c X A}$ is tie proportion of surviving mosquito of age $\mathrm{A}$, that have ever become infected and $V(A) \lambda(A)$ is the proportion of original mosquito that is alive and infected. Let $\mathrm{X}$ denote the proportion of humans who are infectious, and since this is a static analysis, $\mathrm{X}$ is assumes to remain constant. $\mathrm{c}=$ the probability that an uninfected mosquito becomes infected after biting an infectious human. Thus, mosquitoes become infected at rate $a c X$.

iv. Proportion of infectious mosquito $P_{e}=e^{-g n} \mathrm{P}_{\mathrm{e}}=$ the probability of surviving $\mathrm{n}$ days. $\mathrm{n}=$ length of incubation period $\mu(A)=\left(1-e^{-a c X(A-n)}\right) A>n$ the proportion of mosquito of age A that are infectious.

The proportion of mosquito cohort that is alive and infectious at age $\mathrm{A}$ is $V(A) \lambda(A)$. Thus, the proportion of mosquitoes that are infectious, Z, (also called sporozoite), or equivalently the probability that an individual mosquito ever becomes infectious is given by

$$
\int_{0}^{\infty} \beta(A) \lambda(\mathrm{A}) \mathrm{d} \mathrm{A} / \int_{0}^{\infty} \lambda(\mathrm{A}) \mathrm{dA}=\frac{a c X}{\mathrm{~g}+\mathrm{acx}} \mathrm{e}^{-\mathrm{gn}} \quad \ldots \quad \ldots \quad \ldots
$$

Life time transmission potential, according to Smith and Mckenzie [9] is given by

$$
\beta=\int_{0}^{\infty} \operatorname{ba\mu }(A) \lambda(A) d A=\frac{a^{2} b c X e^{-g n}}{\mathrm{~g}(\mathrm{~g}+\mathrm{acX})}
$$

$\mathrm{b}=$ the probability that an uninfected human becomes infected after being bitten by an infectious mosquito.

$\operatorname{ba\mu }(A) \lambda(A)=$ the expected reproductive output of a cohort of mosquito at age A. Life time transmission potential denoted $\beta$, is integrated over a mosquito life time by

$\mathrm{V} \frac{a^{2} b c e^{-g n}}{g^{2}}=\frac{\partial \beta(X, \ldots)}{\partial X}$

$$
\cdots \quad \cdots \quad \cdots
$$

Life time transmission potential for a mosquito is a function of the proportion of a human population that is infectious, $\mathrm{X}$ as well as other parameters. The curve $\beta(\mathrm{X})$ is concave down with slope. Thus, VX $\beta(\mathrm{X})$ the two differ by the factor thus

$$
\left(1+\frac{a c X}{g}\right) e^{-1}\left(\frac{h}{h+a c X}\right)
$$

Note that it is a product of three quantities; Square of the stability index $\left(S^{2}\right)$, the net transmission efficiency (bc), and the probability of surviving the incubation period $\left(\mathrm{P}_{\mathrm{e}}\right)$. The formula $\mathrm{V}$ describes the total contribution of the Victorian capacity of a single mosquito over its life time. Mosquito population can be combined into a single variable $\mathrm{C}(\mathrm{t})$ called Victorian capacity [12]. The average number of bites per individual per day is Ma: Macdonald [3], where M: mosquito density per human, a: average number of human bites per day by any one mosquito. Ross [13], examined the transmission of malaria in population dynamics of human and vector given by

$$
\frac{d y}{d t}=\frac{b^{\prime}}{n} \mathrm{f}^{\prime} \mathrm{y}^{\prime}(\mathrm{n}-\mathrm{y})-(\mathrm{r}+\mathrm{v})
$$

Where; $\mathrm{n}$ : total population sizes of a given time. $\mathrm{y}$ : total number of infected individuals.

f: infected individuals who are not infectious, $r$ : recovery — rate, $\mu$ : birth — rate,

v: death — rate, where prime means the same values with respect to vector.

Dietz [14], developed a transmission dynamics model of malaria defined as,

$$
(1-\delta)^{\mathrm{N}} \mathrm{h}(\mathrm{t}-\mathrm{N}) \mathrm{x}_{1}(\mathrm{t}-\mathrm{N}) \text { i. e. } \mathrm{Q}=(1-\delta)^{\mathrm{N}} \mathrm{h}(\mathrm{t}-\mathrm{N})
$$

Where $\mathrm{t}-\mathrm{N}$ : individual who are infected at time. $\mathrm{x}_{1}(\mathrm{t}-\mathrm{N})$ : proportion of non- immune negative. $\mathrm{h}(\mathrm{t}-\mathrm{N})$ : inoculation rate. $\mathrm{N}$ : proportion of newly infected individual who survive the incubation period of days is approximately $(1-\delta)^{\mathrm{N}}$. Q: total number of infected people.

\section{Materials and Methods}

We used ordinary differential equations to model the growth at each stage from the egg to the adult. The work examines the suitable environmental conditions for various behaviour of anopheles mosquito such as resting, swarming, oviposition, biting and feeding.

To derive the modified model and to establish the stability analysis of the disease free equilibrium states, three methods were used. Firstly, we used the equilibrium point or steady state, then the Beltrami's conditions [15] and finally the method of Diekman's conditions. Maple software was used to show the results when two natural enemies are introduce respectively. 


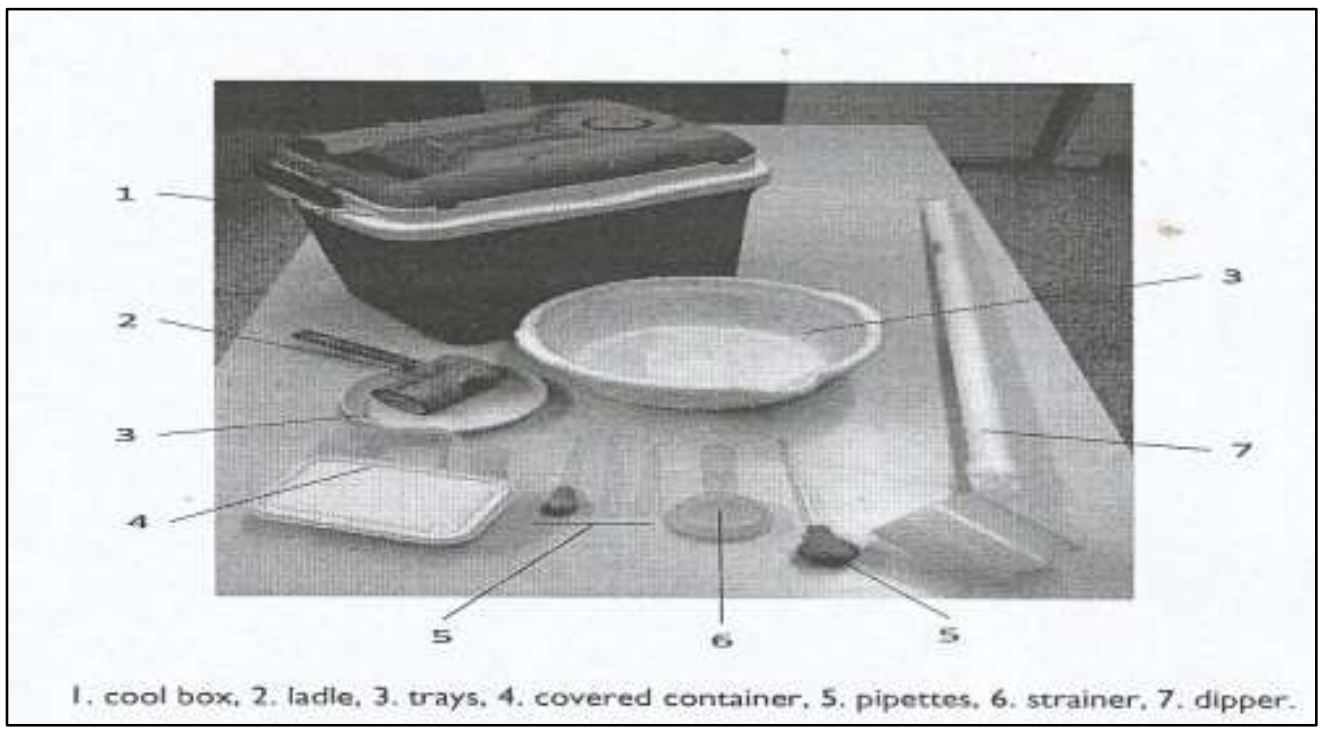

\subsection{Sampling Methods}

Several larval sampling methods are available. The use of each sampling method depends on the nature and type of breeding sites.

The larval collector must approach the breeding site carefully, as any disturbance will cause the larvae and pupae to swim downwards thereby becoming inaccessible. It is important that the collector does not cast a shadow on the water. If the larvae and pupae move, it may be necessary to stand quietly until they swim back up.

\subsection{Dipping Method}

This method is normally used to sample from relatively large water bodies such as swamps, ditches, streams and rice fields. The dipper should be lowered gently at an angle of about $45^{\circ}$ to minimize disruption and either skim the top of the water or gentle lower it to cause the water and nearby larvae to flow into the dipper. Care should be taken not to spill water when raising the dipper from the water. Larvae should be collected from the dipper using a pipette and transferred to a well- labeled bottle or vial. When the breeding site has emerging vegetation, the collector should disturb the water and cause the larvae to swim downwards, then remove some vegetation to create a clear spot for sampling, and wait for a few minutes before resuming sampling as previously described. The number of dips in each breeding place should be annotated for calculation of larval density. Note also the amount of time spent on collection.

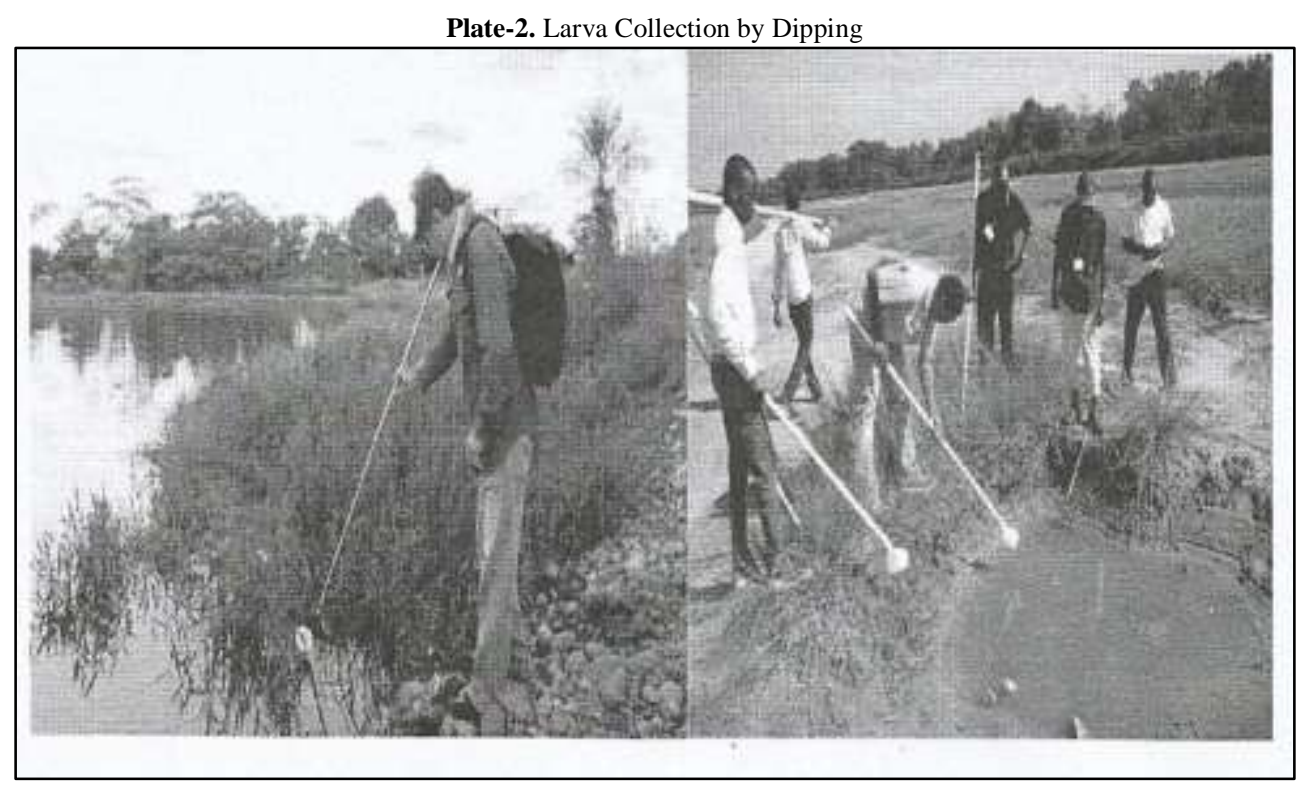

\subsection{Netting Method}

This method consists of using a fine mesh net mounted on a handle, with a plastic bottle or tube tied to one end. It is normally used to collect larvae and pupae in larger water bodies such as ponds and small lakes. The net should be held at about $45^{\circ}$ angle to the water surface and dragged across the surface. The larvae and pupae are collected in the plastic bottle at the end. 


\subsection{Pipetting Method}

This method is used for collecting larvae in small breeding sites such as small puddles, hoof prints, containers, plant axils and tree holes.

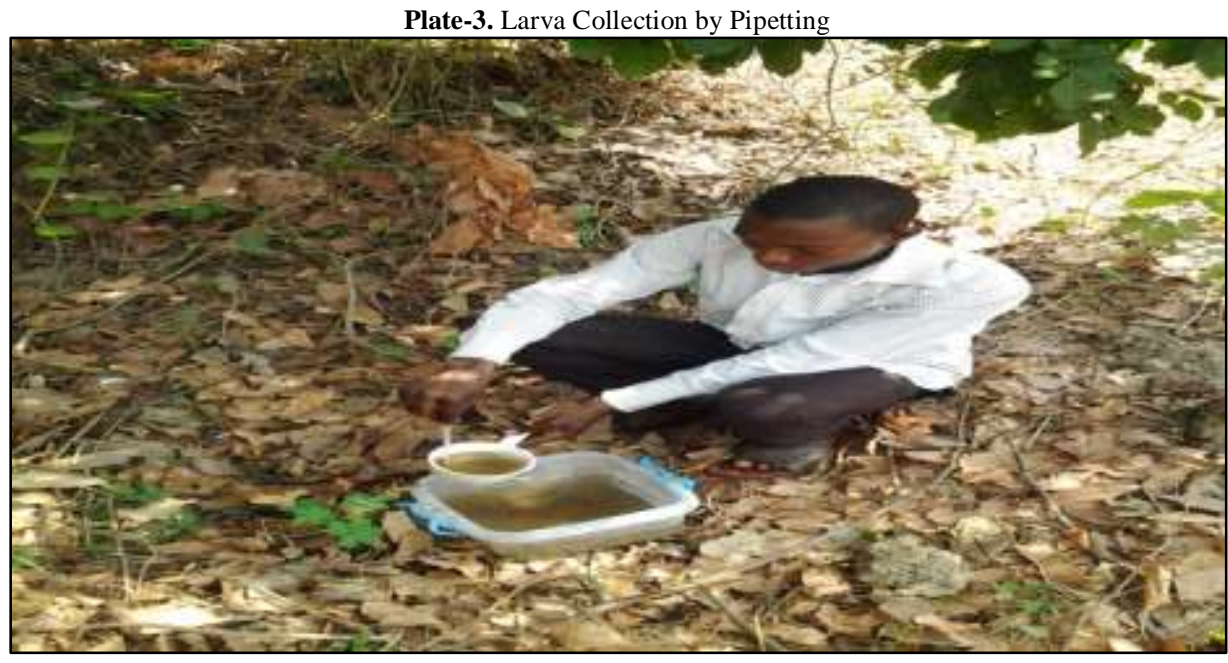

Plate-4. Introduction of Natural Enemy to the Larvae Tray

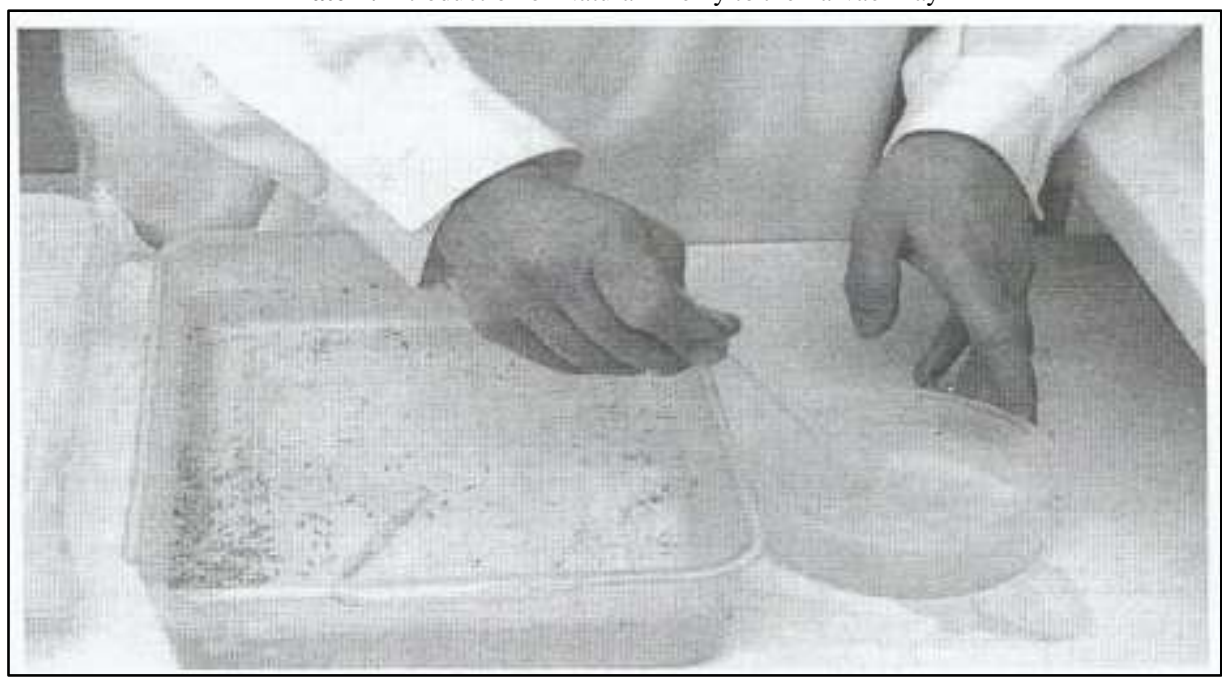

\subsection{Life-cycle of the Anopheles Mosquito}

There are four stages in the life cycle of a mosquito: egg, larva, pupa and adult. During its life-cycle the mosquito undergoes two changes (metamorphoses), from larva to pupa and from pupa to adult.

Plate-5. Stages of the Anopheles Mosquito Life-Cycle

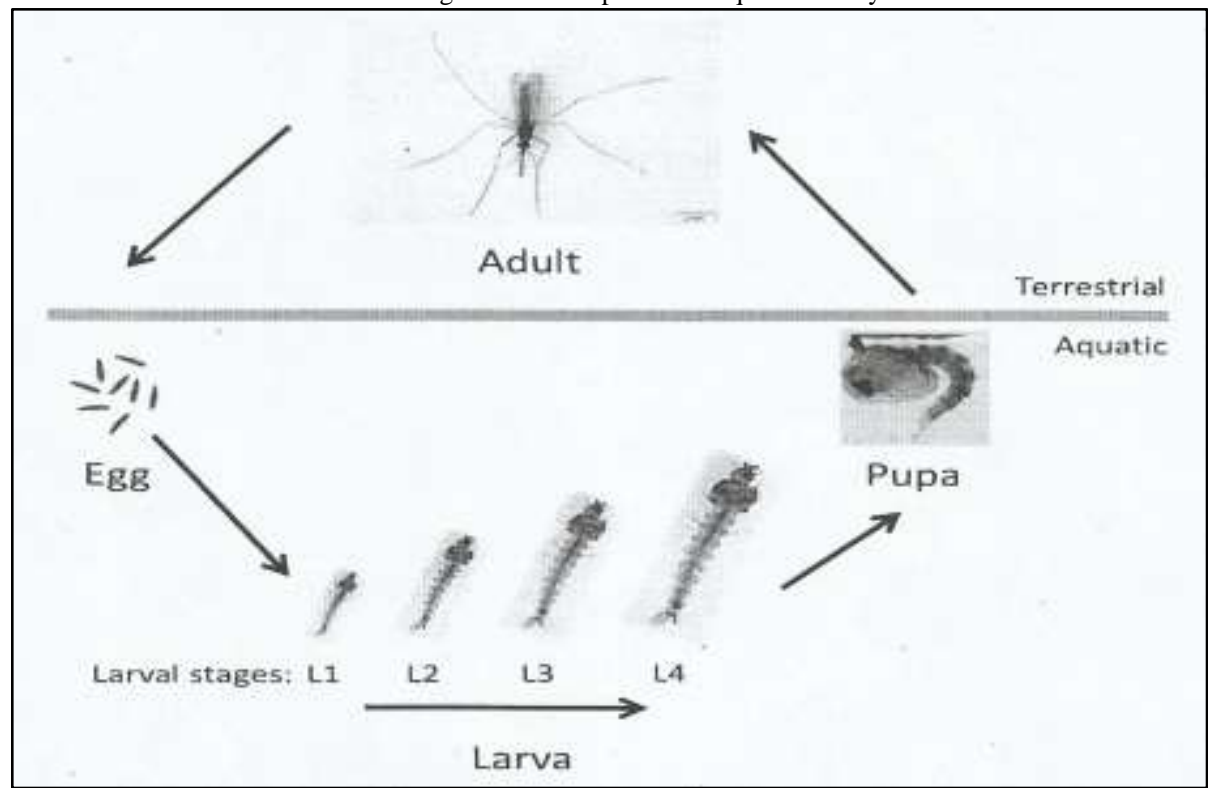




\subsection{Proposed Model Variables and Parameters Defined}

Table-1. Variables and parameters used in new models are defined below

\begin{tabular}{l|l}
\hline Variables & Description \\
\hline $\mathrm{A}(\mathrm{t})$ & Number of adult mosquito at time $(\mathrm{t})$ \\
\hline $\mathrm{B}(\mathrm{t})$ & Number of egg at time $(\mathrm{t})$ \\
\hline $\mathrm{L}(\mathrm{t})$ & Number of larva at time(t) \\
\hline $\mathrm{P}(\mathrm{t})$ & Number of pupa at time $(\mathrm{t})$ \\
\hline $\mathrm{N}(\mathrm{t})$ & Total population \\
\hline $\mathrm{S}_{1}(\mathrm{t})$ & Natural enemy for larva(copepods) \\
\hline $\mathrm{S}_{2}(\mathrm{t})$ & Natural enemy for pupa(tadpoles) \\
\hline Parameters & Description \\
\hline $\mathrm{B}$ & Natural birth rate \\
\hline$\mu$ & Natural death rate \\
\hline$\eta$ & The incidence rate(the rate at which adult mosquitoes oviposit) \\
\hline$\Sigma$ & The proportion at which egg harsh to larva \\
\hline$\Lambda$ & The proportion of larva that transform to pupa \\
\hline A & The rate at which copepods eat up the mosquito larva \\
\hline$\Omega$ & The rate at which tadpoles eat up the mosquito pupa \\
\hline $\mathrm{C}$ & The average temperature of the water culture \\
\hline $\mathrm{B}$ & Probability of larva been eaten up by copepods \\
\hline$\Theta$ & Probability of pupa been eaten up by tadpoles \\
\hline
\end{tabular}

\subsection{Assumptions of the Proposed Model}

The following are the assumptions of the Model

1. The total population of anopheles mosquito consists of four populations.

2. Mosquitoes are given birth to at a rate (b) where the eggs are layed and they die at a rate $(\mu)$ by introducing natural enemies $\mathrm{S}(\mathrm{t})$ to the larva and pupa stage at the rate $\alpha(\mathrm{t})$ and $\omega(\mathrm{t})$.

3. The parasite of one mosquito transferred from one mosquito to the other is only through the medium of host called the horizontal transmission.

4. Emigration and immigration does not occur in this population; however the population increases only through natural birthrate and decreases only through natural death rate.

5. Anopheles mosquito is assumed to transmit malaria only through direct contact.

\subsection{Modeling of Malaria}

Mathematical modeling of malaria is a challenging area of applied mathematics because of its peculiarity in Africa and Nigeria in particular. Millions of people die annually due to malaria. The mosquitoes are resistant to most of the vaccines we have today.

It is of paramount important to develop preventives/methods to convert malaria and mosquito in general.

Figure-1. Flow Diagram of the Adult Anopheles Female Mosquito

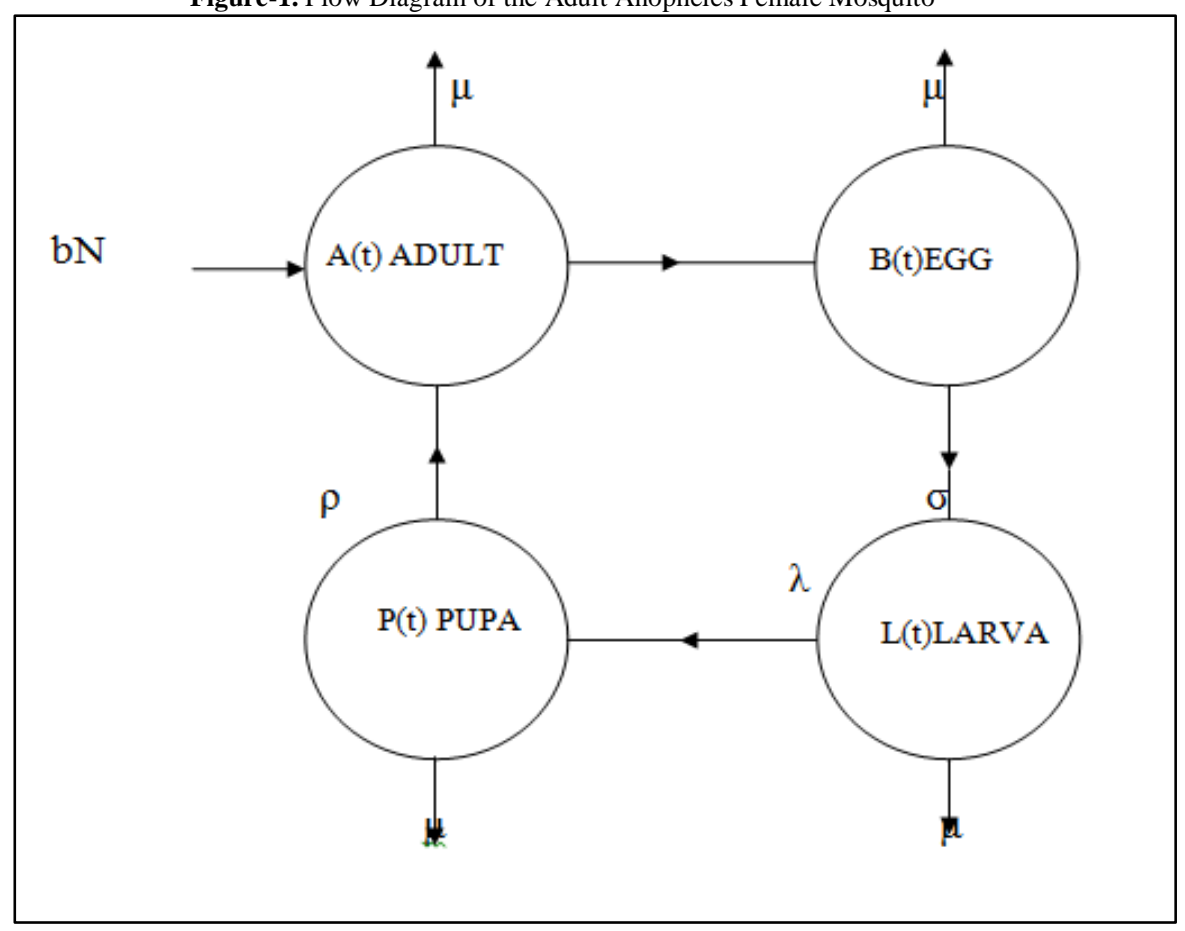




\subsection{The Proposed Model Equations}

$$
\mathrm{N}(\mathrm{t})=\mathrm{A}(\mathrm{t})+\mathrm{B}(\mathrm{t})+\mathrm{L}(\mathrm{t})+\mathrm{P}(\mathrm{t})
$$

$\frac{d A}{d t}=b N-\eta A(t)-\mu A(t)+\rho P(t)$

$\frac{d A}{d t}=b N-(\eta+\mu) A(t)+\rho P(t)$

$\frac{d B}{d t}=\eta A(t)-\sigma B(t)-\mu B(t)$

$\frac{d B}{d t}=\eta A(t)-(\sigma+\mu) B(t)$

$\frac{d L}{d t}=\sigma B(t)+\alpha(t) S(t)-\lambda L(t)-\mu L(t)$

$\frac{d L}{d t}=\sigma B(t)+\alpha(t) S(t)-(\lambda+\mu) L(t)$

$\frac{d P}{d t}=\lambda L(t)-\rho P(t)-\mu P(t)$

$\frac{d P}{d t}=\lambda L(t)-(\rho+\mu) P(t)$

$\mathrm{N}(\mathrm{t})=\mathrm{A}(\mathrm{t})+\mathrm{B}(\mathrm{t})+\mathrm{L}(\mathrm{t})+P(\mathrm{t})$ is the total population of mosquito from egg to adult.

$\mathrm{N}^{\prime}(\mathrm{t})=\mathrm{A}^{\prime}(\mathrm{t})+\mathrm{B}^{\prime}(\mathrm{t})+\mathrm{L}^{\prime}(\mathrm{t})+P^{\prime}(\mathrm{t})$ is the rate of change of the total population of the mosquito from egg to adult.

Natural Enemy: The term "natural enemy" is used for organisms that kill or injure other animals. For example copepods and tadpoles are natural enemy of mosquitoes, predators or parasites are natural enemy of pest insects. Spider are natural enemy of stem borers. Also pathogens are natural enemies. In this work we proposed breaking the life cycle of anopheles mosquito using a natural enemies which is a biological control method for mosquitoes, wherefore, reducing or eliminating the menace of mosquito. we will explain briefly the two forms of natural enemies to be used for the success of this work.

Copepods: Copepods are tiny crustaceans (shrimps, crabs, lobster and relatives) that are wide spread in both fresh and salt water habitat. They are voracious or anxious predators that used to control mosquito production from water holding areas. For effective mosquito control with copepods, knowing where the mosquitoes breed is very essential.

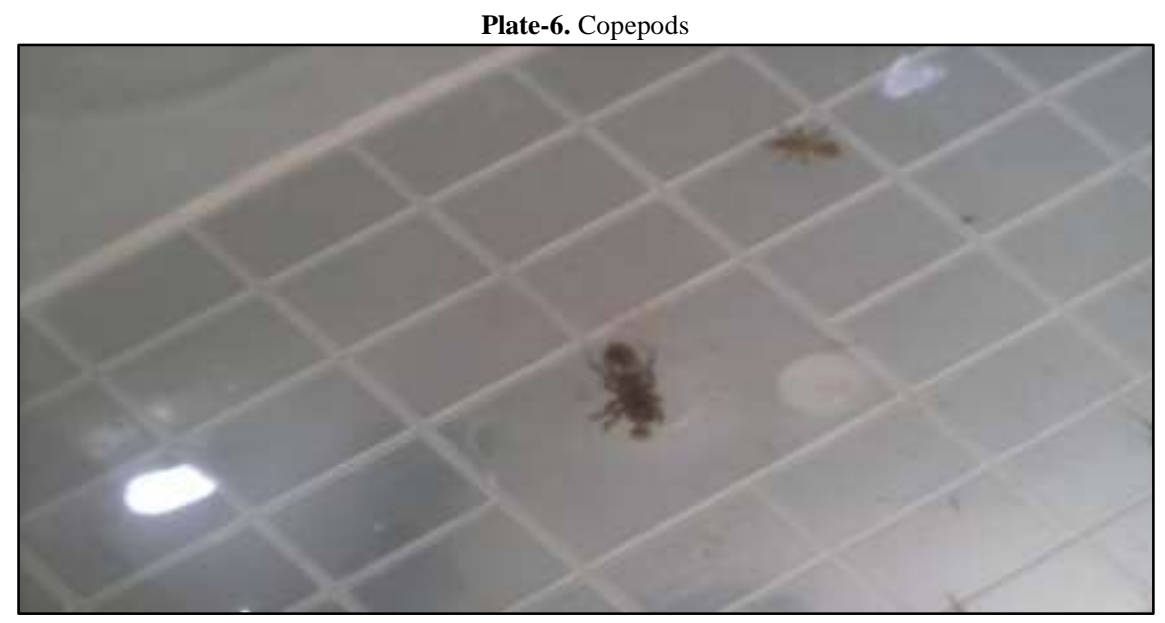

Tadpoles: The aquatic larvae of frogs, toad e.t.c which develops from a limbless tailed form with external gills into a form with internal gills, limbs and reduced tail. They are voracious or anxious predators that used to control mosquito production from water holding areas. For effective mosquito control with tadpoles, knowing where the mosquitoes breed is very essential.

Plate-7. Tadpoles

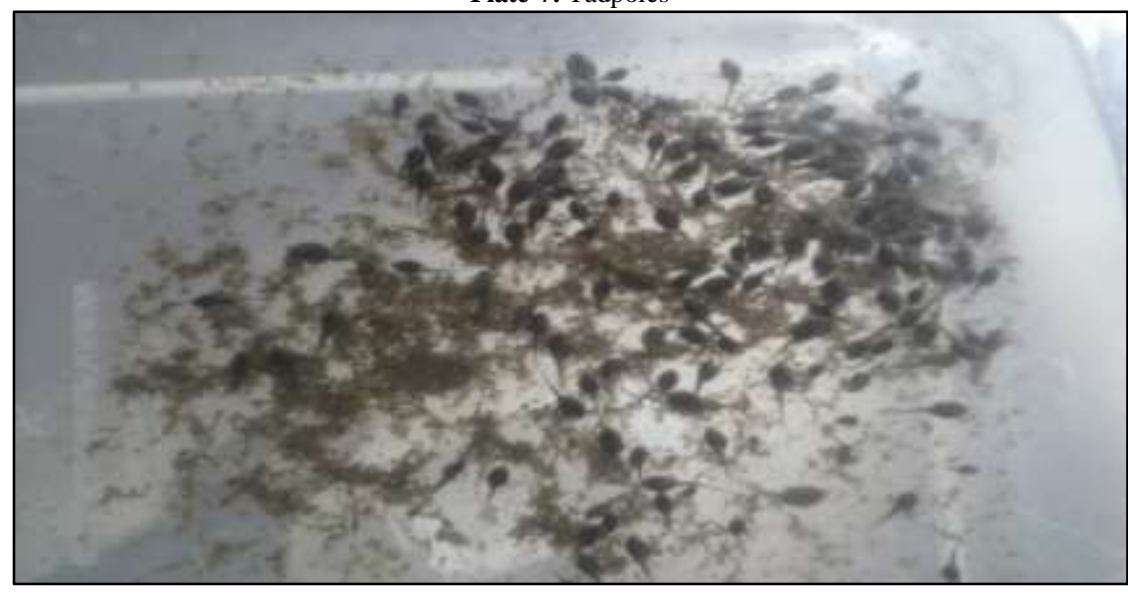


Below is the pictorial Representation of the Modified Model of Equations (11) to (14)

Figure-2. Block Diagram of Adult Anopheles Female Mosquito

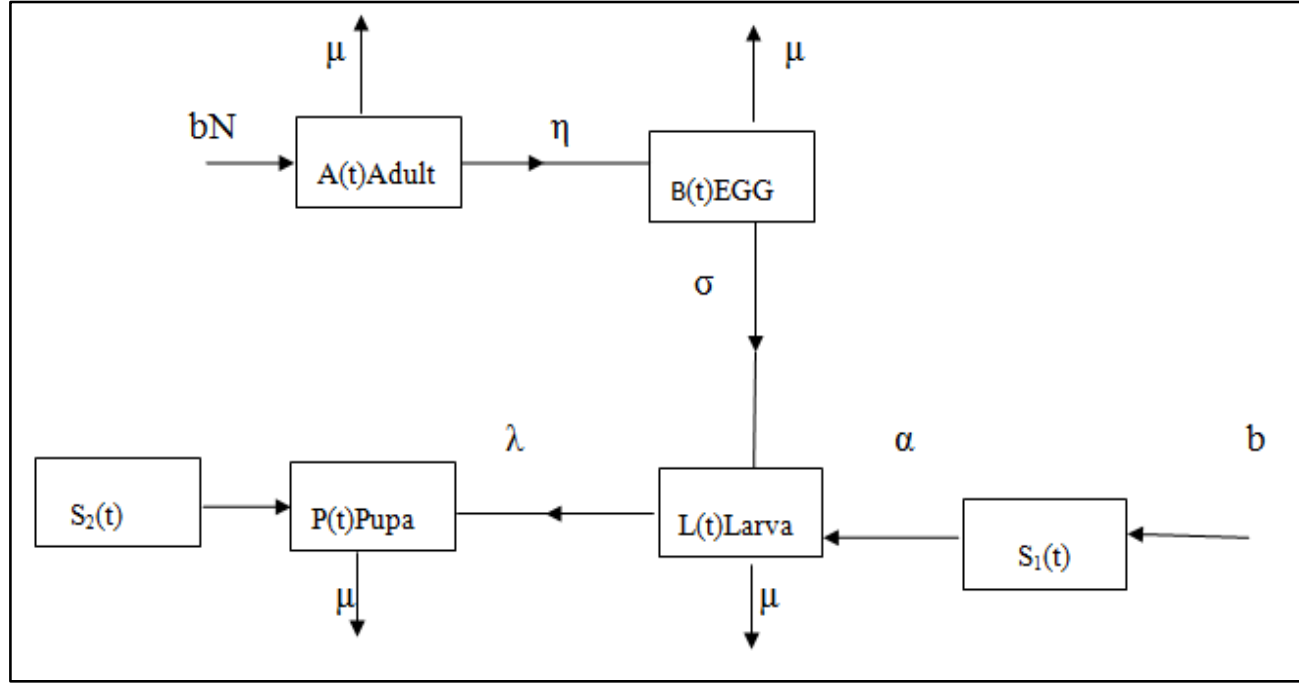

\subsection{Equations of the Modified Model}

$$
\begin{aligned}
& \frac{d A}{d t}=b N-\eta A(t)-\mu A(t) \\
& \frac{d A}{d t}=b N-(\eta+\mu) A(t) \\
& \frac{d B}{d t}=\eta A(t)-\sigma B(t)-\mu B(t) \\
& \frac{d B}{d t}=\eta A(t)-(\sigma+\mu) B(t) \\
& \frac{d L}{d t}=\sigma B(t)+\alpha S 1(t)-\lambda L(t)-\mu L(t) \\
& \frac{d L}{d t}=\sigma B(t)+\alpha S 1(t)-(\lambda+\mu) L(t) \\
& \frac{d P}{d t}=\lambda L(t)+\omega S 2(t)-\mu P(t) \\
& \frac{d P}{d t}=\lambda L(t)+\omega S 2(t)-\mu P(t) \\
& \frac{d S 1}{d t}=b-\alpha S 1(t) \\
& \frac{d S 2}{d t}=b-\omega S 2(t)
\end{aligned}
$$

$\mathrm{N}(\mathrm{t})=\mathrm{A}(\mathrm{t})+\mathrm{B}(\mathrm{t})+\mathrm{L}(\mathrm{t})+P(\mathrm{t})+\mathrm{S}_{1}(\mathrm{t})+\mathrm{S}_{2}(\mathrm{t})$ is the total population of mosquito from egg to adult with natural enemies.

$\mathrm{N}^{\prime}(\mathrm{t})=\mathrm{A}^{\prime}(\mathrm{t})+\mathrm{B}^{1}(\mathrm{t})+\mathrm{L}^{\prime}(\mathrm{t})+P^{\prime}(\mathrm{t})+\mathrm{S}^{1}{ }_{1}(\mathrm{t})+\mathrm{S}^{1}{ }_{2}(\mathrm{t})$ is the rate of change of the total population of the mosquito from egg to adult with natural enemies.

The natural enemies $\mathrm{S}(\mathrm{t})$, is introduced to eat up the larva $\mathrm{L}(\mathrm{t})$ at the rate $\alpha$ and the pupa $\mathrm{P}(\mathrm{t})$ at the rate $\omega$

$$
\text { Where } \alpha=\frac{c \beta S(t)}{L(t)}, \omega=\frac{c \theta S(t)}{P(t)} \text { and } \lambda=1 / \alpha, \rho=0 \text {. }
$$

\section{Analysis and Results}

\subsection{Stability Analysis of the Disease Free Equilibrium States}

Here we would establish the stability of the Model free equilibrium (MFE) state by considering the model parameters and using the model equations. Since we have six systems of non-linear equations or deterministic ordinary differential equation, we know that it is almost impossible to obtain an analytical solution of these systems. Therefore we use the idea of equilibrium point, Beltrami, Diekmann conditions and also use maple software for graphical representation of the result.

\subsection{Equations of the Modified Model}

$$
\begin{aligned}
& \frac{d A}{d t}=b N-(\eta+\mu) A(t) \\
& \frac{d B}{d t}=\eta A(t)-(\sigma+\mu) B(t) \\
& \frac{d L}{d t}=\sigma B(t)+\alpha S 1(t)-(\lambda+\mu) L(t) \\
& \frac{d P}{d t}=\lambda L(t)+\omega S 2(t)-\mu P(t) \\
& \frac{d S 1}{d t}=b-\alpha S 1(t) \\
& \frac{d S 2}{d t}=b-\omega S 2(t)
\end{aligned}
$$




\subsection{Stability Analysis of the Disease Free Equilibrium State (Eo) of the Model}

Let $\mathrm{E}_{0}=\left(\mathrm{A}_{0}, \mathrm{~B}_{0}, \mathrm{~L}_{0}, \mathrm{P}_{0}, \mathrm{~S}_{1}\right.$ and $\left.\mathrm{S}_{2}\right)$ be the equilibrium points of the model (21) to (26). Using equations (21) to (26) we obtain the equations (27) to (32) respectively

$$
\begin{aligned}
& \frac{d A}{d t}=b N-(\eta+\mu) A(t) \\
& \mathrm{bN}-(\eta+\mu)\left(\frac{b N}{\mu+\eta}\right)=0 \\
& \frac{d B}{d t}=\eta A(t)-(\sigma+\mu) B(t) \\
& \eta \mathrm{A}(\mathrm{t})-(\sigma+\mu)\left(\frac{\eta A}{\mu+\sigma}\right)=0 \\
& \frac{d L}{d t}=\sigma B(t)+\alpha S 1(t)-(\lambda+\mu) L(t) \\
& \sigma \mathrm{B}(\mathrm{t})+\alpha(\mathrm{t}) \mathrm{S}_{1}(\mathrm{t})-(\lambda+\mu)\left(\frac{\sigma B+\alpha S 1}{\mu+\lambda}\right)=0 \\
& \frac{d P}{d t}=\lambda L(t)+\omega S 2(t)-\mu P(t), \lambda \mathrm{L}(\mathrm{t})+\omega \mathrm{S}_{2}(\mathrm{t})-\mu\left(\frac{\lambda L+\omega S 2}{\mu}\right)=0 \\
& \frac{d S 1}{d t}=b-\alpha S 1(t) ; \quad \mathrm{b}-\alpha \mathrm{S}_{1}\left(\frac{b}{\alpha \mathrm{S} 1}\right)=0 \\
& \frac{d S 2}{d t}=b-\omega S 2(t) ; \mathrm{b}-\omega \mathrm{S}_{2}\left(\frac{b}{\omega \mathrm{S} 2}\right)=0 \\
& \mathrm{E}_{0}=\left(\frac{b N}{\mu+\eta}, \frac{\eta A}{\mu+\sigma}, \frac{\sigma B+\alpha S 1}{\mu+\lambda}, \frac{\lambda L+\omega s 2}{\mu}, \frac{b}{\alpha \mathrm{S} 1} \text { and } \frac{b}{\omega \mathrm{S} 2}\right)
\end{aligned}
$$

Jacobian Matrix of the Equilibrium Point (Eo) Associated with equations (21) to (26) is

$$
J_{E_{0}}=\left[\begin{array}{cccccc}
\frac{b N}{\mu+\eta} & 0 & 0 & 0 & 0 & 0 \\
0 & \frac{\eta A}{\mu+\sigma} & 0 & 0 & 0 & 0 \\
0 & 0 & \frac{\sigma B+\alpha S 1}{\mu+\lambda} & 0 & 0 & 0 \\
0 & 0 & 0 & \frac{\lambda L+\omega S 2}{\mu} & 0 & 0 \\
0 & 0 & 0 & 0 & \frac{b}{\alpha S 1} & 0 \\
0 & 0 & 0 & 0 & 0 & \frac{b}{\omega S 2}
\end{array}\right]
$$

\subsection{Result for Equilibrium Point (Eo)}

$\mathrm{E}_{0}=\left(\frac{b N}{\mu+\eta}, \frac{\eta A}{\mu+\sigma}, \frac{\sigma B+\alpha S 1}{\mu+\lambda}, \frac{\lambda L+\omega S 2}{\mu}, \frac{b}{\alpha \mathrm{S} 1}\right.$ and $\left.\frac{b}{\omega \mathrm{S} 2}\right)$.This implies that the stability analysis of the disease free equilibrium state using equilibrium point or steady state is stable and the stability of the models means, there will not be anopheles adult mosquito for transmission of malaria parasite in our society.

\subsection{Stability Analysis of the Disease Free Equilibrium State Using Beltrami's Conditions}

Betrami's conditions state that, If the determinants of the Jacobian matrix is greater than zero and the trace elements of the Jacobian matrix is less than zero, then the stability analysis of the free equilibrium state of the model is stable otherwise unstable.

\subsection{Jacobian Matrix (J) Associated with Model Equation is}

$$
J=\left[\begin{array}{cccccc}
-(\eta+\mu) & 0 & 0 & 0 & 0 & 0 \\
\eta & -(\sigma+\mu) & 0 & 0 & 0 & 0 \\
0 & \sigma & -(\lambda+\mu) & 0 & \alpha & 0 \\
0 & 0 & \lambda & -\mu & 0 & \omega \\
0 & 0 & 0 & 0 & -\alpha & 0 \\
0 & 0 & 0 & 0 & 0 & -\omega
\end{array}\right]
$$

\subsection{Contact Matrix (M) Associated with Model Equation is}

$$
M=\left[\begin{array}{cccc}
-(\lambda+\mu) & 0 & \alpha & 0 \\
\lambda & -\mu & 0 & \omega \\
0 & 0 & -\alpha & 0 \\
0 & 0 & 0 & -\omega
\end{array}\right]
$$


Since all the parameters are positive them

$$
\begin{gathered}
\operatorname{Determinant}(m)=(-\alpha) \times(-\omega) \\
=(\alpha)(\omega)
\end{gathered}
$$

\section{$\operatorname{Determinant}(m)>0$}

Now the trace of the contact matrix is the sum of the diagonal of the contact matrix

$$
\begin{aligned}
\operatorname{Trace}(m) & =[-(\lambda+\mu)]+[-(\mu)]+[(-\alpha)]+[(-\omega)] \\
& =-[(\lambda+\mu)+(\mu)+(\alpha)+(\omega)]
\end{aligned}
$$

Since all the parameters are positive them it implies that trace $M<0$

\subsection{Result for Beltrami's Conditions}

Since the determinant of the Contact matrix is greater than zero and the trace of the contact matrix is less than zero, then we conclude that the stability analysis of the disease free equilibrium state of the model is stable, which showed that the rate of malaria parasite in our society will be reduce.

\subsection{Stability Analysis of the Disease Free Equilibrium State Using Diekmann's Conditions}

Diekmann conditions state that, if the Basic reproduction number is less than one $(R o<1)$, then the stability analysis of the free equilibrium state is stable otherwise unstable.

We re-categories equations (27) - (32) into two classes for stability analysis as follows:

$N(t)=A(t), B(t), P(t),(\alpha+\omega) S(t)$

$X=[A(t), B(t)]$ and $Y=[L(t), P(t), S(t)]$

$X=[A(t), B(t)]=b N-(\mu+\eta) A(t)+\eta A(t)-(\sigma+\mu) B(t)$

$=b N-\mu A(t)-(\sigma+\mu) B(t)$

$=b N-\sigma X-2 X$

$=b N-(2+\sigma) X$

$Y=[L(t), P(t), S(t)]=\sigma B(t)+\alpha S 1(t)-(\lambda+\mu) L(t)+\lambda L(t)+\omega S 2(t)-\mu P(t)+b-\alpha S 1(t)+b-\omega S 2(t)$

$=\sigma B(t)-\mu L(t)-\mu P(t)+2 b$

$=\sigma X-\mu Y-\mu Y+2 b$

$=\sigma X-2 \mu Y+2 b$

$$
\text { let; } H=\frac{\delta H}{\delta Y}=-2 \mu
$$

But; $\mathrm{H}=\mathrm{M}-\mathrm{D}, \mathrm{M}=0, \mathrm{D}>0$, where $\mathrm{D}$ is a diagonal matrix then we have the basic reproduction number as;

$$
R o=F\left(M D^{-1}\right)=\frac{M}{D}=\frac{0}{2 \mu} \text { and } \quad R o=0<1
$$

\subsection{Result for Diekmann Conditions}

Since Ro $<1$, by Diekmann conditions, the stability analysis of the free equilibrium state is stable. Since $\alpha=\frac{C \beta S 1(t)}{L(t)}, \omega=\frac{C \beta S 2(t)}{P(t)}$ and $\lambda=\frac{1}{\alpha}, \rho=0$ implies that when $\alpha$ and $\omega \rightarrow \infty$, then $\lambda \rightarrow 0$ hence, the larger the rate of natural enemy that eat up the mosquito larva and pupa, the smaller the proportion of mosquito larva that will be transform to pupa and pupa to adult is zero. With the natural implication, there will not be anopheles adult mosquito for malaria transmission in our society.

\subsection{Numerical Experiment of the Model}

Table-2. Variables and parameters Values

\begin{tabular}{l|l}
\hline Variables/Parameters & Values \\
\hline $\mathrm{A}$ & 50 \\
\hline $\mathrm{B}$ & 25000 \\
\hline $\mathrm{L}$ & 20000 \\
\hline $\mathrm{P}$ & 10000 \\
\hline $\mathrm{B}$ & 0.002 \\
\hline$\mu$ & 0.5 \\
\hline $\mathrm{N}$ & 55250 \\
\hline $\mathrm{S}_{1}$ & 200 \\
\hline $\mathrm{S}_{2}$ & 200 \\
\hline $\mathrm{H}$ & 0.002 \\
\hline$\Sigma$ & 0.00004 \\
\hline$\Lambda$ & 0.00005 \\
\hline $\mathrm{A}$ & 100 \\
\hline$\Omega$ & 50 \\
\hline
\end{tabular}

\subsection{List of Numerical Experiments}

The following experiments were carried out.

1. Effect of introducing two natural enemies on adult anopheles female mosquito. 
2. Effect of introducing two natural enemies on the egg, larva and pupa stage.

3. Effect of introducing two natural enemies on both the larva and the pupa stage.

\subsection{Graphical Presentation of the Results}

Figure-3. Number of Adult Anopheles Female Mosquito, when two natural enemies (copepods and tadpoles) are introduced respectively. $\left(\mathrm{S}_{1}=200, \mathrm{~S}_{2}=200, \alpha=100\right.$ and $\left.\omega=50\right)$

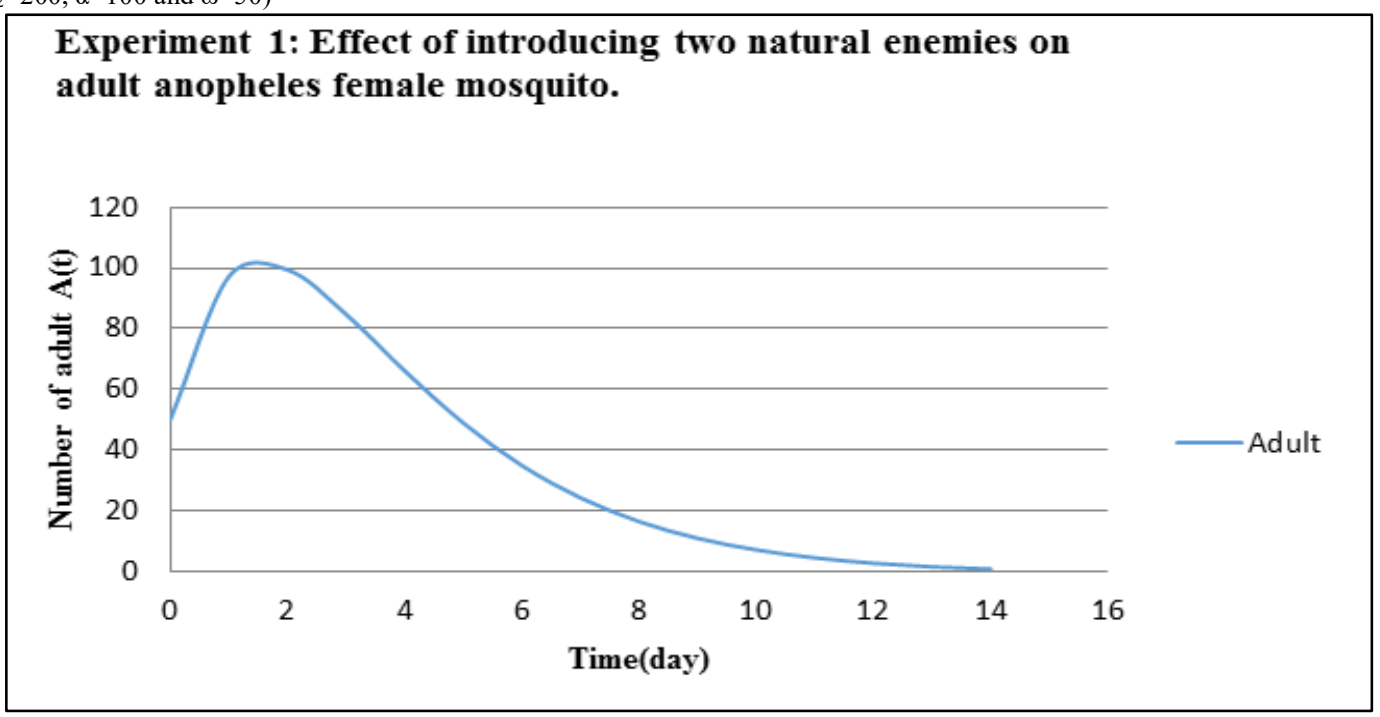

Figure-4. Number of egg, larva and pupa, when two natural enemies (copepods and tadpoles) are introduced respectively $\left(S_{2}=200, S_{1}=200, \alpha=100\right.$ and $\omega=50)$

\section{Experiment 2: Effect of introducing two natural enemies on egg, larva and pupa stage.}

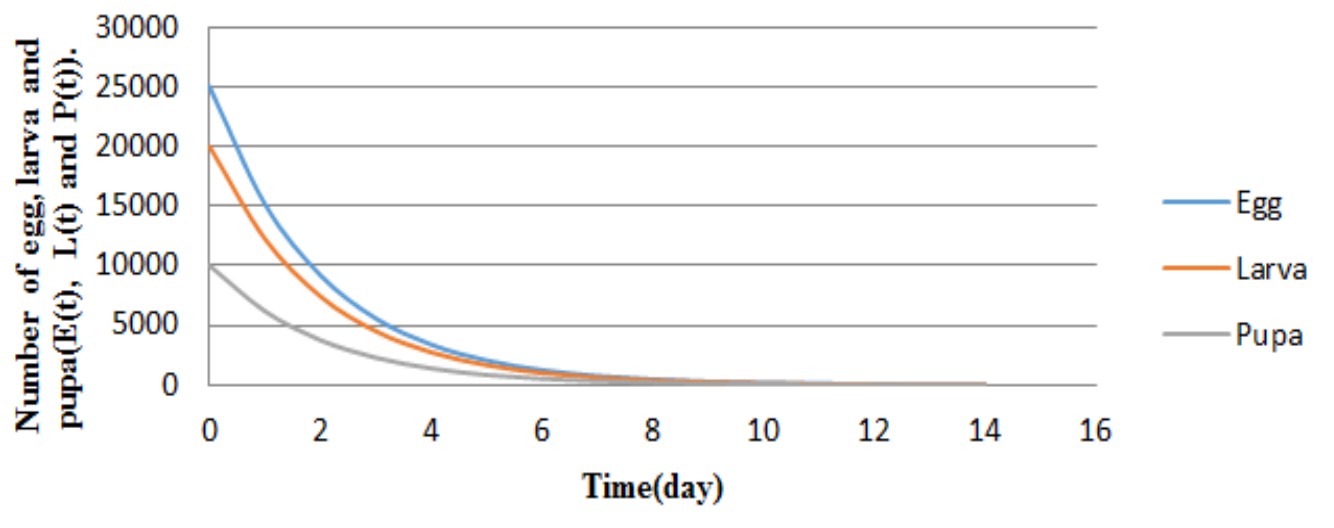

Figure-5. Number of larva and pupa, when two natural enemies (copepods and tadpoles) are introduced respectively ( $S_{1}=200, S_{2}=200, \alpha=100$ and $\omega=50)$

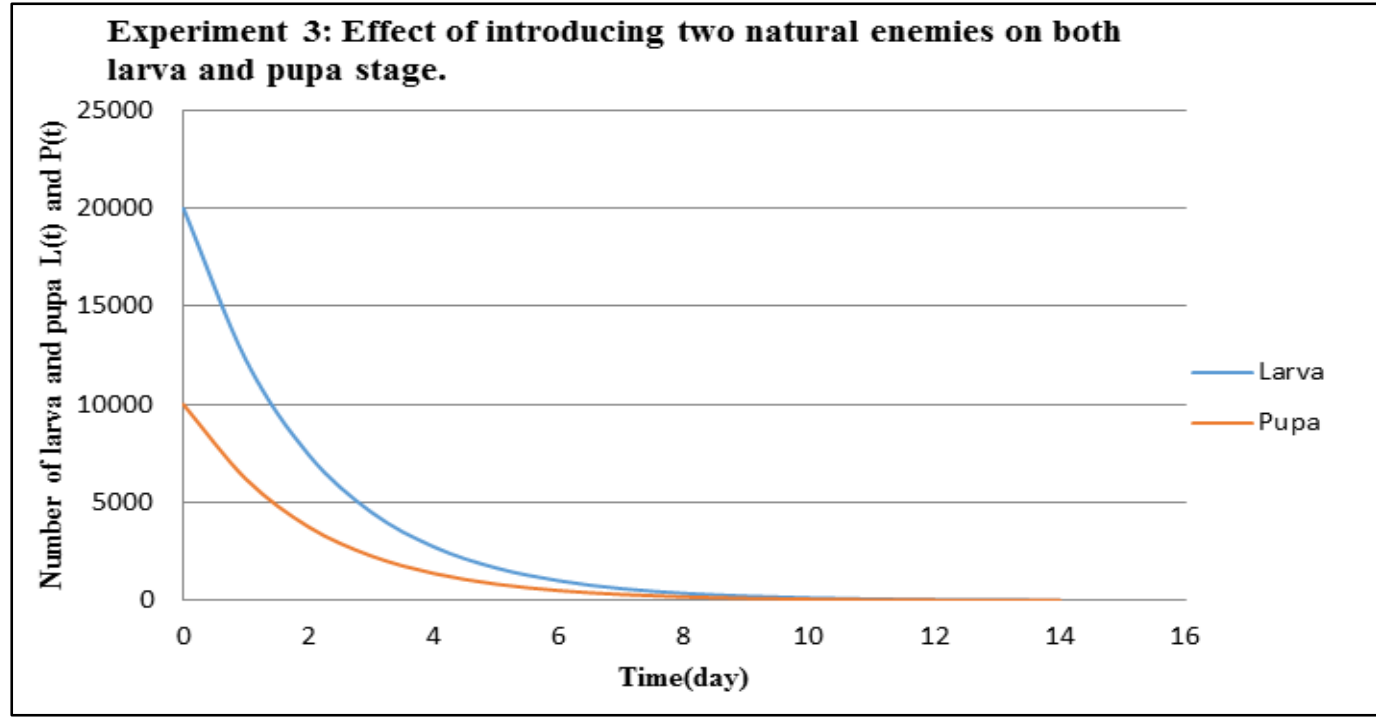




\section{Discussion Conclusion and Recommendation \\ 5.1. Discussion of Results}

A mathematical model to break the life cycle of anopheles mosquito using copepods at larva stage and tadpoles at pupa stage is presented. We discuss the prevalence of mosquito in our society, where two million deaths is attributed to malaria parasite in Sub-Saharan Africa in general and Nigeria in particular out of which one third are children. We discussed the developmental stages of anopheles mosquito which is the egg, larva, pupa and adult. We made mention of the conditions that breed the mosquito, mosquitoes breed faster in the area of high humidity. In chapter two we reviewed related models to this research work. We discussed the materials and methods used, defined the model parameters and variables, made assumptions, represented the model pictorially, derived the model equations and introduced two natural enemies (copepods and tadpoles) to the model at larva and pupa stage. We established the stability analysis of the disease free equilibrium state of the model using the idea of equilibrium point, Beltrami's conditions, Diekmann conditions and we also use maple software for symbolical and numerical solution and present the results graphically, which showed the effect of introducing two natural enemies (copepods and tadpoles) on both larva and the pupa stage. From the result we observed that the stability analysis of the free equilibrium state is stable. With the natural implication, there will not be anopheles adult female mosquito for malaria transmission in our society. The new model used parameters as shown in Table 1. These parameters are chosen with the threshold values obtained in the stability analysis of the disease free equilibrium state of the model. In the analytic result, the model analysis revealed the existence of a unique disease free equilibrium $\operatorname{state}\left(E_{0}\right)$, which is locally and asymptotically stable when $R_{0}<1$. We also identify the basic reproduction number, $R_{0}$ in terms of the model parameters. In the numerical results, numerical experiments carried out using parameter values in Table 1 and applying the conditions for stability of the disease free equilibrium state $\left(E_{0}\right)$ gives the following result: In Experiment 1, the effect of introducing two natural enemies (copepods and tadpoles) on adult anopheles female mosquito was investigated as shown in Figure 1, when two natural enemies are introduced respectively. The infection in the adult anopheles mosquito population is slow down significantly and consequently eradicated, and the probability of transmission in the adult anopheles female mosquito population is very low.

In Experiment 2, the effect of introducing two natural enemies (copepods and tadpoles) on egg, larva and pupa was investigated as shown in Figure 2, when two natural enemies are introduced respectively. Result shows that, the rate of infection reduce sharply in Figure 2. To prevent new malaria infection the rate of transmission must be reduced to near zero, hence in recent years prevention strategies has moved to the top in the fight against malaria. This has led to a number of global efforts to develop new technologies to prevent malaria as recorded by Yau [16].

Considering the entire population, the effect of introducing two natural enemies (copepods and tadpoles) was investigated when two natural enemies are introduced respectively, (Compare Figure 1 and Figure 2 with Figures 3, 4 and 5). When two natural enemies are introduced the proportion of infective is drastically reduced and the infection eradicated in the egg, larva and pupa, but persists in small proportion in the adult anopheles mosquito.

Finally to understand the impact of introducing two natural enemies (copepods and tadpoles) in the adult, egg, larva and pupa when two natural enemies are introduced respectively, graphical illustrations are given in Figure 1,2 and 3. It was clearly observed that reducing the rate of transmission to the barest minimum. This could be achieved as research focus should be on formulating models that captures preventive strategies base on stability analysis to prevent the incidence of the disease and consequently eradicate the disease.

\section{Conclusion}

We observed that based on the conditions of Beltrami's which state, if the determinants of the Jacobian matrix is greater than zero and the trace is less than zero are satisfied then the stability analysis of the disease free equilibrium state is stable and Diekmann conditions which says if $R_{0}<1$ the stability analysis of the disease free equilibrium state is stable. We thereby conclude that when the natural enemy introduced is much then the number of larva leading to pupa will be almost zero and the number of pupa develop to adult will be zero and that will break the life-cycle of the anopheles mosquito. Hence, there will not be anopheles adult mosquito for transmission of malaria parasite in our society.

\section{References}

[1] Coetzee, M., Craig, M., and Le Sueur, D., 2000. "Distribution of african malaria mosquitoes belonging to the anopheles gambiae complex." Parasitol Today, vol. 16, pp. 74-77.

[2] Killeen, G. F., Mckenzie, F. E., Foy, B. D., Schieffelin, C., Billingslay, P. F., and Beier, J. C., 2000. "A simplified model for predicting malaria entomologic inoculation rates based on entomologic parameters relevant to control." Am. J. Trop. Hyg., vol. 62, pp. 535-544.

[3] Macdonald, G., 1957. The epidemiology and control of Malaria. London: Oxford University press.

[4] Benedict, M., 2009. Methods in anopheles research. Malaria Research and Reference Reagent Center. Version, pp. 264-270.

[5] WHO, 1975. Manual on practical entomology in malaria. Geneva Switzerland: World Health Organization. p. 160.

[6] WHO, 2005. Guidelines for laboratory and field testing of long-lasting insecticidal mosquito nets. Geneva, Switzerland: World Health Organization. WHO/CDS/WHOPES/GCDPP. p. 11.

[7] WHO, 2006. "Malaria vector control and personal protection. World healthorganization technical report series, no. 936, Geneva, Switzerland." p. 6. 
[8] WHO, 2009. "2008 world malaria report. World health organization. Geneva, Switzerland.Who/htm/gmp/2008:." pp. 1-29.

[9] Smith, D. L. and Mckenzie, F. E., 2004. "Statics and dynamics of malaria infections in anopheles mosquitoes." Malaria Journal, vol. 3, Available: https://doi.org./10.1186/1475-2875-3-13

[10] Jepson, W. F., Moutia, A., and Courtois, C., 1947. "The malaria problem in mauritius: The bionomics of mauritian anophelines." Bull Entomol Res., vol. 38, pp. 177-208.

[11] Diekmann, O., Heesterbeek, J. A. P., and Metz, J. A. J., 1990. "On the definition and computation of the basic reproduction ratio ro in models for infectious disease of heterogeneous populations." J. Math. Biol., vol. 28 , pp. $365-382$.

[12] Garret, J. C., 1964. "Prognosis for interruption of malaria transmission through assessment of the mosquitoes vectorial capacity." Nature, vol. 204, pp. 1173-1175.

[13] Ross, R., 1981. The prevention of Malaria. London John Murray.

[14] Dietz, K., 1993. "The estimation of the basic reproduction number for infectious disease." Stat. Methods Med. Res., vol. 2, pp. 23-41.

[15] Beltrami, E., 1989. Mathematics for dynamic modeling. N.Y: Academic Press. p. 203.

[16] Yau, M., 2011. "A mathematical model to break the life cycle of mosquitoes." Shiraz E medical Journal, vol. 12, pp. 121-127.

\section{Appendix 1}

Maple Command for Symbolical Solution, When two Natural Enemies are introduced.

sol1 $:=\operatorname{dsolve}([\operatorname{diff}(\mathrm{A}(\mathrm{t}), \mathrm{t})=.2 \mathrm{e}-2 * \mathrm{~N}(\mathrm{t})-.502 * \mathrm{~A}(\mathrm{t})$,

$\operatorname{diff}(\mathrm{B}(\mathrm{t}), \mathrm{t})=.2 \mathrm{e}-2 * \mathrm{~A}(\mathrm{t})-.50004 * \mathrm{~B}(\mathrm{t})$

$\operatorname{diff}(\mathrm{L}(\mathrm{t}), \mathrm{t})=.4 \mathrm{e}-4 * \mathrm{~B}(\mathrm{t})+100 * \mathrm{~S} 1(\mathrm{t})-.50005 * \mathrm{~L}(\mathrm{t})$

$\operatorname{diff}(\mathrm{N}(\mathrm{t}), \mathrm{t})=.2 \mathrm{e}-2 * \mathrm{~N}(\mathrm{t})-.4 \mathrm{e}-2-.5 * \mathrm{~A}(\mathrm{t})-.5 * \mathrm{~B}(\mathrm{t})-.5 * \mathrm{~L}(\mathrm{t})-.5 * \mathrm{P}(\mathrm{t})$,

$\operatorname{diff}(\mathrm{P}(\mathrm{t}), \mathrm{t})=.5 \mathrm{e}-4 * \mathrm{~L}(\mathrm{t})+50 * \mathrm{~S} 2(\mathrm{t})-.5 * \mathrm{P}(\mathrm{t})$,

$\operatorname{diff}(\mathrm{S} 1(\mathrm{t}), \mathrm{t})=.2 \mathrm{e}-2-100 * \mathrm{~S} 1(\mathrm{t})$,

$\operatorname{diff}(\mathrm{S} 2(\mathrm{t}), \mathrm{t})=.2 \mathrm{e}-2-50 * \mathrm{~S} 2(\mathrm{t})$,

$\mathrm{A}(0)=50, \mathrm{~B}(0)=25000, \mathrm{~L}(0)=20000, \mathrm{~N}(0)=55050, \mathrm{P}(0)=10000, \mathrm{~S} 1(0)=200, \mathrm{~S} 2(0)=200])$;

$\operatorname{plot}([2116903317211740524 / 76347225449001 * \exp (-249 / 500 * \mathrm{t})-$

$715594509784788492 / 25856408433667 * \exp (-251 / 500 * \mathrm{t})-2 / 62499 * \mathrm{t}+3333333 / 165004166600 * \exp (-$

$100 * \mathrm{t})+4999999 / 61256249900 * \exp (-50 * \mathrm{t})-$

$1249904812103 / 781225000200,105845165860587026200 / 3893708497899051 * \exp (-$

$249 / 500 * \mathrm{t})+35779725489239424600 / 1266964013249683 * \exp (-251 / 500 * \mathrm{t})-100 / 781299999 * \mathrm{t}-$

$5208145022033201 / 813906251249866668-3333333 / 8208953988266668 * \exp (-100 * \mathrm{t})-$

$4999999 / 1516090959900002 * \exp (-50 * \mathrm{t})-36574808473916539454325272500 / 1202163500421224191202499 * \exp (-$ $12501 / 25000 * \mathrm{t}),-80 / 7813781289999 * \mathrm{t}+406941736362597137290199 / 101758630079897394229033335$ -

$146299233895666157817301090000 / 1202163500421224191202499 * \exp (-$

$12501 / 25000 * \mathrm{t})+84676132688469620960 / 159642048413861091 * \exp (-249 / 500 * \mathrm{t})-$

$9541260130463846560 / 16470532172245879 * \exp (-251 / 500 * \mathrm{t})-$

$1368158861228544861111089 / 6806587594873617105555^{*} \exp (-$

$100 * \mathrm{t})+9999998 / 3752321335525105199995 * \exp (-$

$50 * \mathrm{t})+4969413251509368834407176000 / 35009057816782740773511 * \exp (-10001 / 20000 * \mathrm{t})$,

$2116903317211740524 / 159642048413861091 * \exp (-249 / 500 * \mathrm{t})+238531503261596164 / 16470532172245879 * \exp (-$ $251 / 500 * \mathrm{t})+6875170156927361111111 / 68065875948736171055550000 * \exp (-100 * \mathrm{t})-$

$3790222813192765752499000001 / 18761606677625525999975000 * \exp (-$

$50 * \mathrm{t})+4070752144934342041309289999 / 1017586300798973942290333350000-$

$1 / 976722661249875 * \mathrm{t}+182874042369582697271626362500 / 1202163500421224191202499 * \exp (-12501 / 25000 * \mathrm{t})-$ $4969413251509368834407176000 / 35009057816782740773511 * \exp (-10001 / 20000 * \mathrm{t})], \mathrm{t}=0 . .10$, color $=[\mathrm{red}$, green, yellow, black]);

\section{Appendix 2}

\begin{tabular}{|c|c|c|c|c|c|c|c|c|c|c|c|c|c|c|c|}
\hline & \multicolumn{14}{|c|}{ EFFECT OF INTRODUCE TWO NATURAL ENEMIES. } & \\
\hline $\mathrm{t}$ & 0 & 1 & 2 & 3 & 4 & 5 & 6 & 7 & 8 & 9 & 10 & 11 & 12 & 13 & 14 \\
\hline A & 50 & 97.22026 & 99.41659 & 84.70946 & 66.06078 & 48.84919 & 34.83089 & 24.15759 & 16.36723 & 10.84352 & 7.008805 & 4.39911 & 2621961 & 1.42023 & 0.660776 \\
\hline$B$ & 25000 & 15162.79 & 9196.486 & 5577.873 & 3383.133 & 2051.981 & 1244,604 & 754.9056 & 457.8861 & 277.7316 & 168.4596 & 102.1805 & 61.97844 & 37.59338 & 22.80219 \\
\hline L & 20000 & 12253.14 & 7431.903 & 4507.676 & 2734.044 & 1658.282 & 1005.8 & 610.0499 & 370.0153 & 224.4271 & 136.1234 & 82.56457 & 50.07949 & 30.37631 & 18.42573 \\
\hline$N$ & 55250 & 33501.59 & 20281.65 & 12247.29 & 7364.49 & 4396.923 & 2593.421 & 1497.349 & 831.2132 & 426.3695 & 180.3246 & 30.78898 & -60.0937 & -115.33 & -148.903 \\
\hline$p$ & 10000 & 6188.452 & 3753.859 & 2277.058 & 1381.244 & 837.851 & 508.2342 & 308.2917 & 187.0084 & 113.4392 & 68.8127 & 41.74272 & 25.32232 & 15.36185 & 9.31993 \\
\hline$\$ 1$ & 200 & $4.00 E-05$ & $4.00 E-05$ & $4.00 E-05$ & $4.00 E-05$ & 4.00E-05 & $4.00 \mathrm{E}-05$ & $4.00 E-15$ & 4.00E- -05 & $4.00 E-05$ & 4.00E-05 & $4.00 E-05$ & $4.00 E-05$ & $4,00 E-05$ & $4.00 \mathrm{E}-05$ \\
\hline$\$ 2$ & 200 & 4.00E-05 & $4.00 E-05$ & $4.00 E-05$ & 4.00E- -05 & 4.00E- -05 & $4,00 E-05$ & $4.00 E-05$ & $4.00 E-05$ & 4.00E- 05 & 4.00E- -05 & $4.00 E-05$ & $4.00 E-05$ & $4.00 E-05$ & $4.00 E-05$ \\
\hline
\end{tabular}




\section{Appendix 3}

Symbolical Solution Using Maple Software, when two Natural Enemies are introduced

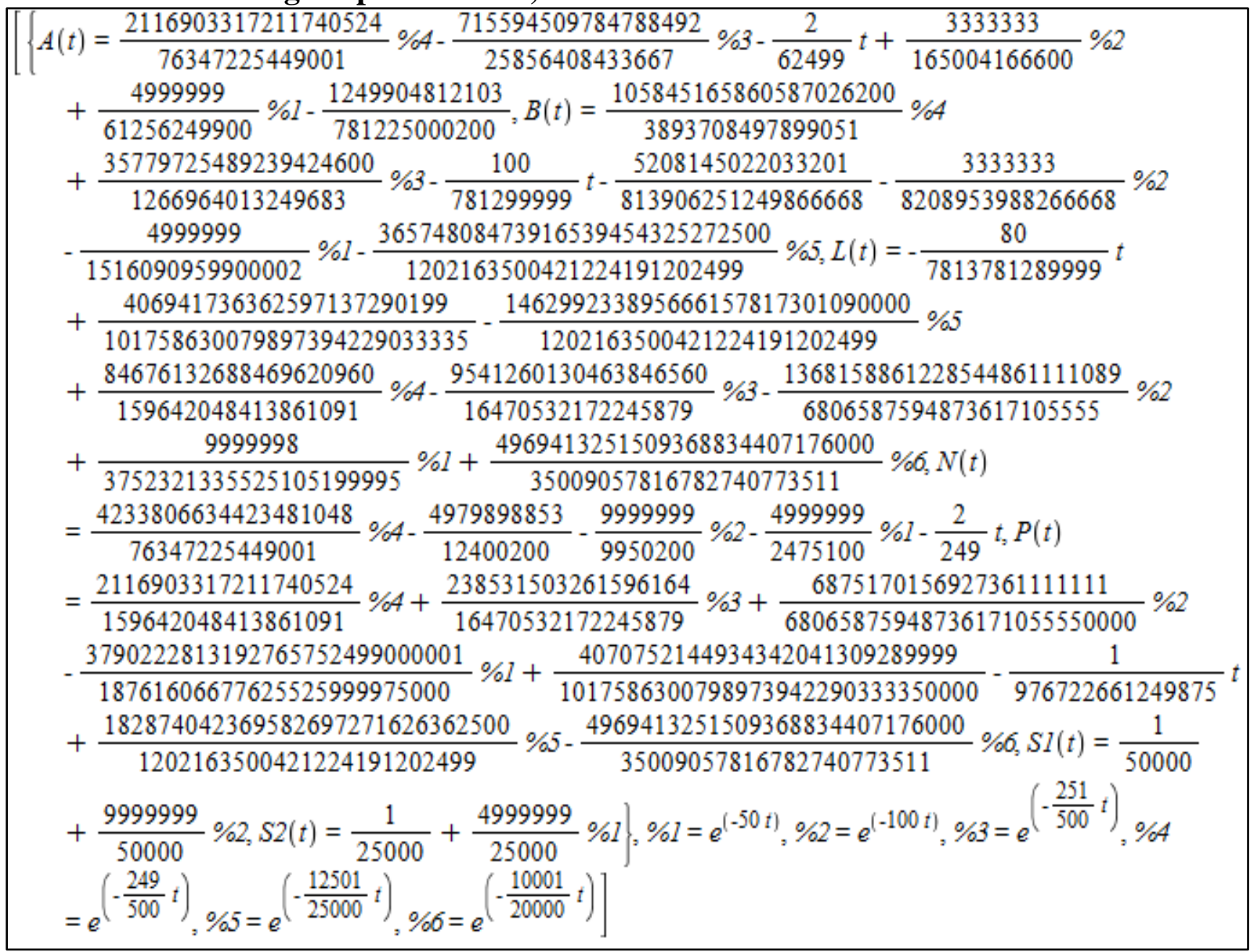

